\title{
The 50s Cliff: Perceptuo-Motor Learning Rates across the Lifespan
}

\author{
Rachel O. Coats ${ }^{1 *}$, Andrew D. Wilson ${ }^{2}$, Winona Snapp-Childs ${ }^{3}$, Aaron J. Fath ${ }^{3}$, Geoffrey P. Bingham ${ }^{3}$
}

1 School of Biomedical Sciences, Faculty of Biological Sciences, University of Leeds, Leeds, United Kingdom, 2 School of Social, Psychological and Communication Sciences, Leeds Metropolitan University, Leeds, United Kingdom, 3 Department of Psychological and Brain Sciences, Indiana University, Bloomington, Indiana, United States of America

\begin{abstract}
We recently found that older adults show reduced learning rates when learning a new pattern of coordinated rhythmic movement. The purpose of this study was to extend that finding by examining the performance of all ages across the lifespan from the $20 \mathrm{~s}$ through to the $80 \mathrm{~s}$ to determine how learning rates change with age. We tested whether adults could learn to produce a novel coordinated rhythmic movement $\left(90^{\circ}\right.$ relative phase) in a visually guided unimanual task. We determined learning rates to quantify changes in learning with age and to determine at what ages the changes occur. We found, as before, that learning rates of participants in their $70 \mathrm{~s}$ and $80 \mathrm{~s}$ were half those of participants in their $20 \mathrm{~s}$. We also found a gradual slow decline in learning rate with age until approximately age 50 , when there was a sudden drop to a reduced learning rate for the 60 though 80 year olds. We discuss possible causes for the " $50 \mathrm{~s}$ cliff" in perceptuo-motor learning rates and suggest that age related deficits in perception of complex motions may be the key to understanding this result.
\end{abstract}

Citation: Coats RO, Wilson AD, Snapp-Childs W, Fath AJ, Bingham GP (2014) The 50s Cliff: Perceptuo-Motor Learning Rates across the Lifespan. PLoS ONE 9(1): e85758. doi:10.1371/journal.pone.0085758

Editor: Natasha M. Maurits, University Medical Center Groningen UMCG, Netherlands

Received October 4, 2013; Accepted December 2, 2013; Published January 24, 2014

Copyright: (c) 2014 Coats et al. This is an open-access article distributed under the terms of the Creative Commons Attribution License, which permits unrestricted use, distribution, and reproduction in any medium, provided the original author and source are credited.

Funding: The authors have no support or funding to report.

Competing Interests: The authors have declared that no competing interests exist.

*E-mail: r.o.a.coats@leeds.ac.uk

\section{Introduction}

The coordination of perception and action is intrinsic to numerous tasks of daily living such as walking, eating, dressing and driving a vehicle. Coordination is of primary concern to older adults because the loss of the ability to produce smooth coordinated muscle activity will lead to an increased risk of falling [1] and a decreased social and functional independence [2]. Perceptuo-motor learning, as well as performance, is essential for retaining independence, maintaining health or recovering from injury, and reducing the burden on caregivers and society at large. Older adults are also often required to re-learn coordination skills after injury, or learn new patterns of coordination such as fastening buttons with one hand or walking with walking sticks. Of course learning coordinative skills is relevant not only to older adults but adults of all ages. New tasks need to be learned on a continuous basis, especially with the advances in technology we are experiencing at the moment, and anyone can suffer from an injury that results in the need to re-learn previously attained coordinative skills. We recently discovered that older adults in their $70 \mathrm{~s}$ and $80 \mathrm{~s}$ learned a new pattern of coordination at half the rate of a group of 20 year olds [3]. Here we investigated the rest of the lifespan between these ages to determine how learning rates change with age.

Coordinated rhythmic movement (first described by Kelso, [4]) is a useful way of assessing perceptuo-motor learning. When two limbs, or two oscillators of any sort, move in phase (e.g. when two fingers move upwards and downwards at the same time) they are said to move at $0^{\circ}$ relative phase. When they move in opposition (or anti-phase) they are at $180^{\circ}$ relative phase. $0^{\circ}$ is the most stable form of coordination. $180^{\circ}$ can also be readily produced, but is less stable than $0^{\circ}$ with people spontaneously switching to $0^{\circ}$ at high frequencies. Both $0^{\circ}$ and $180^{\circ}$ can be produced with little intent or conscious effort [5]. They are also said to represent the intrinsic dynamics of the system and do not require learning [6]. Other coordination patterns such as $90^{\circ}$ are difficult to produce in the absence of special circumstances (e.g. following metronomes) or training [5-8]. Wilson et al [11] showed that, without special training and perceptual learning, $90^{\circ}$ relative phase is not perceived well and discriminated from different relative phases (other than $0^{\circ}$ and $180^{\circ}$ ). Thus, it is not surprising that $90^{\circ}$ relative phase is not easy to envisage. Imagine two oscillators/dots moving from left to right on a computer screen, one above the other. For $0^{\circ}$ the dots move together - one always directly above the other and in the same direction (See Figure 1). For $180^{\circ}$ the dots move in opposite directions. So when one moves left the other moves right and vice versa. In $90^{\circ}$ (halfway between $0^{\circ}$ and $180^{\circ}$ ) the dots are moving in the same direction only $50 \%$ of the time (see Figure S1). When the dots move from left to right one dot reaches the right side at the same time as the other one reaches the midpoint at peak speed. Many people see the pattern as one dot 'chasing the other' and always staying half the amplitude of the movement behind it. Due to the fact that it is hard to produce without training, we used the $90^{\circ}$ relative phase pattern in the current experiment because we wanted to examine how people across the lifespan cope with learning a novel task; akin to the idea of a person with osteoarthritis learning to walk with a cane or a patient who has suffered from a bad fall or had a hip replacement re-learning to 

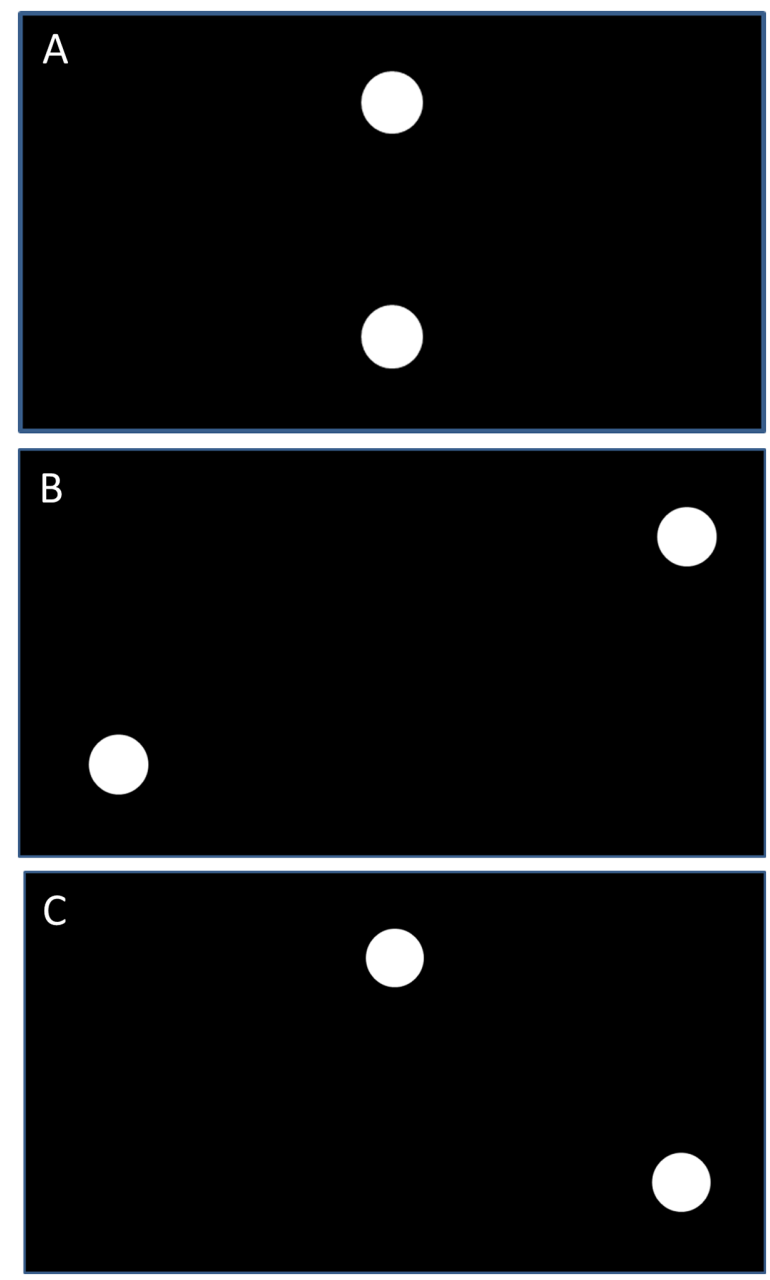

Figure 1. $0^{\circ}, 180^{\circ}$ and $90^{\circ}$ degrees. The oscillators at the three phase relations: $0^{\circ}$ (a), $180^{\circ}$ (b) and $90^{\circ}$ (c). doi:10.1371/journal.pone.0085758.g001

walk properly. It's not whether they can learn to produce 90 per se, but whether they can produce a new pattern or coordination.

Perceptual information is considered a crucial element of successful coordination [9-11]. In visual coordination studies where only vision is available to couple movements, and participants oscillate their limb to coordinate with the movement of another oscillator on a computer display [12-13], or another person [14-15], all the usual coordination dynamics are exhibited showing that coordination is mediated by perception. From this perception-action perspective on coordination it follows that rhythmic production of $90^{\circ}$ is difficult because participants are unable to perceive and recognise it. This is not to say that observers cannot learn to perceive and produce it with training, they can $[11,16]$, but stable production requires stable access to perceptual information and this needs to be learned. Additional evidence for the central role of perception comes from studies using lissajous displays (position-position plots), where transformed visual feedback information leads to improved performance of $90^{\circ}$ relative phase [17-18] and studies where $90^{\circ}$ performance is possible when the perceived phase relation displayed is transformed to look like $0^{\circ}[13,19]$.

Most research into perceptuo-motor performance and learning has been carried out on young adults. Less is known about older adults, and even less about those between these two age groups.
Serrien et al. [7] examined bimanual performance of $0^{\circ}$ and $180^{\circ}$ relative phase in young (mean age 24 ) and older (mean age 75 ) adults and found performance deteriorated for the older adults when dissimilar limbs were used, and this was enhanced in the anti-phase mode. Swinnen et al [6] investigated the performance of bimanual $90^{\circ}$ rhythmic movements with lissajous feedback by nine older (mean age 73) and nine younger (mean age 19) participants. They found that older adults showed lower performance levels than the younger adults across acquisition and retention and were more variable. In respect to learning, the younger adults showed a steep decline in error on day 1 , but this decrease was equivalent to that of the older adults on day 2. Wishart et al. [20] also examined bimanual performance and learning of $90^{\circ}$ by older and young adults with lissajous feedback. Both groups could learn to produce $90^{\circ}$, although the older adults were not as consistent as the younger adults, and only benefitted from concurrent visual feedback at the end of day 3, whereas the younger adults benefitted on day 1 .

In a recent study, Coats et al [3] sought to fill a gap in the existing literature by quantifying learning rates and changes in learning rates. They examined unimanual/visual coordination without transformed feedback in older and younger adults. They found that older adults exhibited learning rates that were half those of their younger counterparts and suggested that, while there are likely to be a number of factors underlying these age differences, emerging deficits in motion perception may be key. In the current study, we set out to determine how and when this change in learning rate occurs by examining the rest of the lifespan. Is there a gradual change in performance with age? Or is there a sudden drop, and if so at what age? Participants followed the exact same procedure as those in Coats et al [3]. 9-10 participants from each age decade from $20 \mathrm{~s}$ to $80 \mathrm{~s}$ were tested on a visual coordination task with concurrent feedback on performance. Participants were tested on their baseline ability to move at $0^{\circ}, 90^{\circ}$, and $180^{\circ}$, trained at $90^{\circ}$ over 5 sessions, and re-evaluated at post test and retention. The data yielded learning curves that were fit by a model and used to estimate learning rates, separately for each of the age groups. The majority of the data from the 20, 70 and 80 year olds have already been published [3] so we would expect the same learning rates in these groups (with rates of the older groups being half that of the younger group), but how learning rates change across the lifespan $(30 \mathrm{~s}, 40 \mathrm{~s}, 50 \mathrm{~s}, 60 \mathrm{~s}$ in addition to $20 \mathrm{~s}, 70 \mathrm{~s}$, and $80 \mathrm{~s}$ ) remains to be seen.

\section{Method}

\section{Ethics Statement}

This study was approved by the Institutional Review Board at IU Bloomington. Written informed consent was obtained from all participants.

\section{Participants}

Ten young adult participants in their $20 \mathrm{~s}$ ( 3 male; mean age 22), 10 participants in their $30 \mathrm{~s}$ (5 male; mean age 33), 10 in their $40 \mathrm{~s}$ (3 male; mean age 44), 9 in their $50 \mathrm{~s}$ (2 male; mean age 54), 10 in their $60 \mathrm{~s}$ (8 male; mean age 67), 10 in their $70 \mathrm{~s}$ (3 male; mean age 74) and 9 in their $80 \mathrm{~s}$ (3 male; mean age 84) participated in the study. The majority of participants were recruited from the Indiana University or wider Bloomington community including the YMCA, IU Tennis Center, Meadowood retirement community (six 70 year olds and seven 80 year olds), and from fliers placed around the IU campus. Four of the 60 year olds and one 83 year old were recruited from Sandbach Rugby Club, UK. All participants had normal or corrected-to-normal 
vision. All participants were naïve to the experimental questions and their $90^{\circ}$ relative phase production was worse than their $0^{\circ}$ and $180^{\circ}$ relative phase production prior to training. Measures of cognitive function were collected from the older adults $70 \mathrm{~s}$ and $80 \mathrm{~s})$ using the Short Portable Mental Status Questionnaire [21] and all participants scored within the range of normal mental functioning. Data from all the 20 year olds, nine 70 year olds and eight 80 year olds have already been published [3].

\section{Procedure}

The procedure was identical to that used in Coats et al. [3]. Participants sat in front of a Dell Latitude 15" laptop, with the monitor set to a resolution of $1024 \times 768$ and a refresh rate of $60 \mathrm{~Hz}$ that was connected to a Logitech Force 3D Pro joystick (force feedback feature disabled) via USB. The computer presented a display showing two white dots, one above the other, on a black background. In the display, the dots oscillated horizontally from side-to-side. Except for task demonstrations, the top dot was under the control of the computer, while the bottom dot was under the control of the participant via the joystick. All participants used their preferred hand to control the joystick. The amplitude of movement of each dot was 300 pixels and each dot was 60 pixels in diameter. Stimulus presentation, data recording and all data analysis was handled by a custom Matlab toolbox written by ADW, incorporating the Psychtoolbox (http://psychtoolbox.org) [22-24].

There were four Assessment sessions (Baseline x2, Post Training and Retention) and five Training sessions. These sessions were spread over eight separate days (not necessarily consecutive but within a nine week period). We conducted two baseline sessions primarily to ensure that task novelty was not too large a contributing factor to baseline performance. The measures that we report for "baseline" represent the averaged performance during these two sessions. In the assessment sessions, participants first viewed an $8 \mathrm{~s}$ demonstration of the $0^{\circ}$ target relative phase then they attempted to produce $0^{\circ}$ five times by moving the joystick from side to side. All movement trials were $20 \mathrm{~s}$ in duration. The first trial was practice and online feedback was given. The feedback was a "hot/cold" signal where the dot that the participant controlled turned green within a certain range and is described below and in Wilson et al. [25]. Participants were told prior to the practice trial that when the feedback was "on" during the practice trial then they were moving successfully. During the subsequent trials, no feedback was given (participants were apprised that no feedback would be available during these trials). This procedure was then repeated for the $180^{\circ}$ and $90^{\circ}$ target relative phase conditions.

In each of five training sessions, participants performed ten $20 \mathrm{~s}$ $90^{\circ}$ trials, with an $8 \mathrm{~s}$ demonstration before every trial. So, participants performed a total of 50 trials over five separate days. During each trial, online feedback was provided by changing the colour of the person-controlled dot from white to green when the participant was moving at $90^{\circ},+/-$ an error bandwidth. The error bandwidth was faded (decreased) across sessions when performance reached a certain threshold. The level participants were started on in the first training session was dependent on performance at baseline: data were analysed to see at which error bandwidth (from $+/-35^{\circ}$ to $+/-10^{\circ}$ in $2.5^{\circ}$ intervals) the participant could perform the task (i.e. stay within this bandwidth) $50 \%$ of the time, and this was the level at which they started. After subsequent training sessions, data were again analysed in a similar way, and the error bandwidth was altered for the next training session (but only by a maximum of $5^{\circ}$ each time) if performance improved. Participants were informed of this change. If perfor- mance did not improve the error bandwidth remained the same. Changes to the error bandwidth, which drives learning, were therefore self paced.

\section{Data Analysis}

The two position time series from each trial were filtered using a low-pass Butterworth filter with a cut-off frequency of $10 \mathrm{~Hz}$ and numerically differentiated to yield a velocity time series. These were used to compute a time series of relative phase, the key measure of coordination between the two dots.

To assess the stability of the coordination over the course of a trial, we used a measure of proportion of time on task (PTT). The measure is the proportion of time during a trial that the relative phase falls within $\mathrm{a}+/-20^{\circ}$ window of the target relative phase (e.g. $90^{\circ}$ ). We averaged PTT, for each participant, over the trials performed in a given condition. We chose PTT as the primary measure because, in human movement, stability is not independent of mean relative phase; so measures that simply assess overall movement variability (e.g. the standard deviation of mean relative phase or mean vector length) are confounded with the actual relative phase produced. Coordination stability at $90^{\circ}$ can be artificially elevated if participants spend time at other locations (e.g. $0^{\circ}$ or $180^{\circ}$ ), which they do as these locations are natural attractors [5], (see Wilson et al. [13] for an extended analysis of this problem). Proportion of time on task allows us to address this problem (see Snapp-Childs, et al. [10] for an explicit comparison of the two methods). It is simply the proportion of the relative phase time series that falls within the range of the target phase + / - a tolerance (e.g. of $20^{\circ}$ ), thus summarizing the data of interest (consistency and accuracy) and eliminating the confound. This measure ranges from $0-1$ and validly measures stability of coordination at the required relative phase in a single number $[11,25]$.

We examined the differences between performance pre and post-training at $0^{\circ}, 90^{\circ}$ and $180^{\circ}$ for all age groups using mixed design ANOVAs with session (baseline, post-test and retention) and relative phase $\left(0^{\circ}, 180^{\circ}\right.$ and $\left.90^{\circ}\right)$ as repeated measures factors, and group (20-year olds, 30-year olds, 40 year-olds, 50 year-olds, 60 year-olds, 70-year olds, 80-year olds) as a between-subjects factor. Further mixed ANOVAs (group $\times$ session) were used to look at relative phase separately, and paired t-test/one-way ANOVAs utilised to examine interactions.

We also examined learning rate. Exponential functions were fitted to the data. The functions were of the form:

$$
P T T \equiv a * \exp (-b / s)
$$

where PTT is 'Proportion of Time on Task', $\mathrm{S}$ is session $(1=$ baseline and $7=$ post-training), and $\mathrm{a}$ and $\mathrm{b}$ are parameters. The function was fitted in three different ways and results were compared to be sure they were essentially the same. First, the function was fit to the means separately for each age group using Quasi-Newton estimation in Systat 5.2. Secondly, the PTT means and session numbers were transformed as follows:

$$
P T T \rightarrow \ln (P T T) \text { and } S \rightarrow 1 / S
$$

Least squares linear regression was used to fit a line to the relation between the two sets of transformed values, again separately for each group. Third and finally, transformation and linear regression was used again applied to the combined 
individual participant data for each group, However, in this last case, we also used multiple linear regression to test differences in slope and intercept between the groups taken two at a time. Once values for $\mathrm{a}$ and $\mathrm{b}$ parameters were identified (and judged to be equivalent) we computed the first derivative of the function in Equation (1) and evaluated it at Session 1 to get a value for learning rate.

\section{Results}

Figure 2 shows the performance of all groups at baseline, posttest and retention for all conditions, measured as proportion of time-on-task (PTT) using a $+/-20^{\circ}$ window. The figure shows that all groups performed equally poorly at baseline for the $90^{\circ}$ pattern, and any group differences at $0^{\circ}$ and $180^{\circ}$ at baseline appear small if present at all. All groups were better at performing $0^{\circ}$ than $180^{\circ}$ in all three sessions. For the $90^{\circ}$ pattern, it is evident that the younger groups $(20 \mathrm{~s}, 30 \mathrm{~s}, 40 \mathrm{~s}$ and $50 \mathrm{~s})$ showed a greater improvement between baseline and post-test than the older groups $(60 \mathrm{~s}, 70 \mathrm{~s}$ and $80 \mathrm{~s})$.

We performed 3-way mixed design ANOVA with session (baseline, post-test and retention) and relative phase $\left(0^{\circ}, 180^{\circ}\right.$ and $90^{\circ}$ ) as repeated measures factors, and group (20-year olds, 30year olds, 40 year-olds, 50 year-olds, 60 year-olds, 70-year olds, 80 -year olds) as a between-subjects factor. Group was significant
$\left[\mathrm{F}(6,61)=5.25 ; \mathrm{p}<0.01, \eta_{\mathrm{p}}{ }^{2}=0.34\right]$, with the 20,30 and 40 year olds (mean time-on-task $=0.58,0.58$ and 0.59 respectively) performing better than the 50 and 60 year olds (mean timeon-task $=0.50$ for both), who were better than the 70 and 80 year olds (mean time-on-task $=0.46$ and 0.45 ). A significant main effect of session was also identified $\left[\mathrm{F}(2,122)=55.37 ; \mathrm{p}<0.001, \eta_{\mathrm{p}}{ }^{2}=\right.$ 0.48 ], with performance at post-training (mean time-on-task $=0.54$ ) and retention (mean time-on-task $=0.55$ ) being better than performance at baseline (mean time-on-task $=0.47$ ). Relative phase was significant $\left[\mathrm{F}(2,122)=217.9 ; \mathrm{p}<0.001, \eta_{\mathrm{p}}{ }^{2}=0.78\right]$ with performance at $0^{\circ}$ (mean time-on-task $=0.69$ ) being better than performance at $180^{\circ}$ (mean time-on-task $=0.52$ ) and $90^{\circ}$ (mean time-on-task $=0.36)$. Significant interactions were found between session and relative phase $[\mathrm{F}(4,244)=36.24 ; \mathrm{p}<0.001$, $\left.\eta_{\mathrm{p}}{ }^{2}=0.37\right]$, but not between session and group or relative phase and group. The 3-way interaction between group, session and relative phase was also significant $[\mathrm{F}(24,244)=2.73 ; \mathrm{p}<0.01$, $\left.\eta_{\mathrm{p}}{ }^{2}=0.21\right]$ so further analyses were required to reveal the nature of this interaction.

\section{$90^{\circ}$}

First, we wanted to determine whether all groups could learn the $90^{\circ}$ relative phase pattern. Although all groups seem to improve between baseline and post-test, it is clear that the younger participants $(20 \mathrm{~s}-50 \mathrm{~s})$ show a larger increase in time-on-task
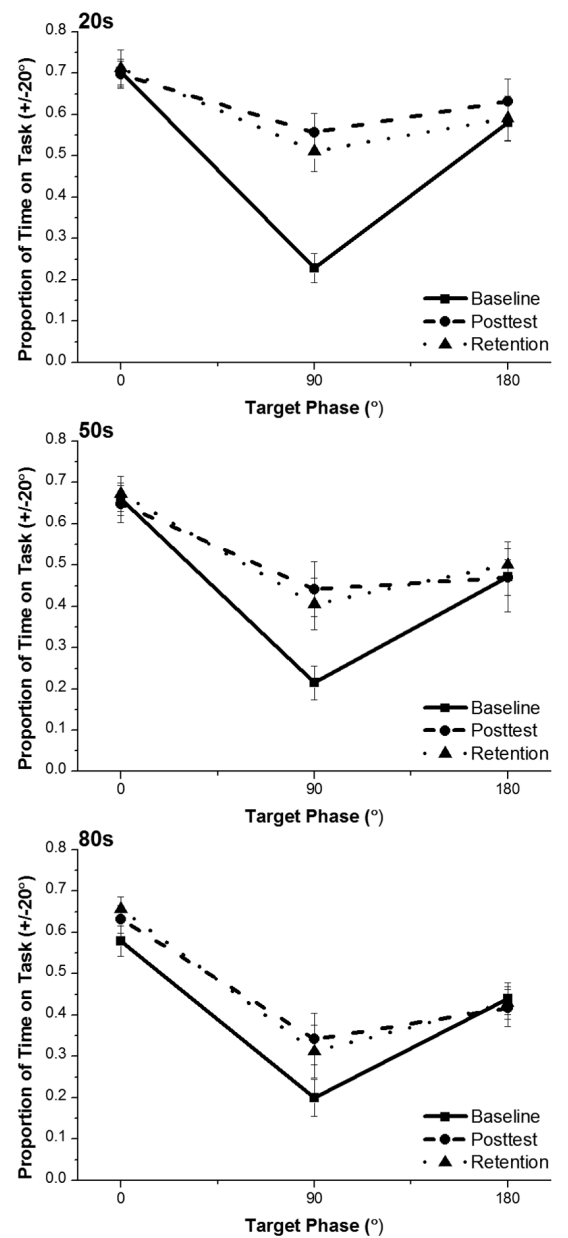
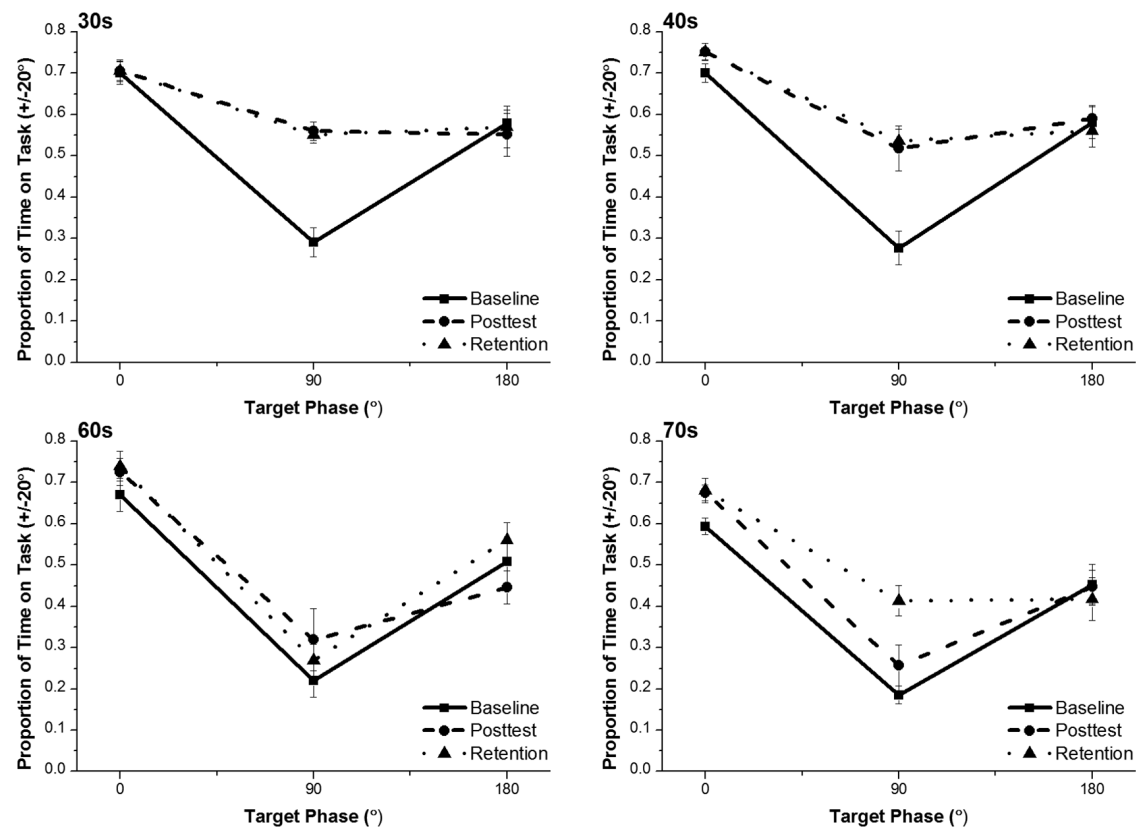

Figure 2. Proportion of time on task across age, relative phase, and assessment session. Proportion of time spent within $20^{\circ}$ of the target mean relative phase $\left(0^{\circ}, 90^{\circ}\right.$, or $180^{\circ}$ ) across the baseline (solid line), post-training (dash-dot line) and retention (dotted line) sessions for all age groups. doi:10.1371/journal.pone.0085758.g002 
between baseline and post-test than the older participants $(60 \mathrm{~s}-$ 80s). A repeated measures ANOVA on the $90^{\circ}$ performance data at baseline, post-test and retention revealed a significant main effect of group $\left[\mathrm{F}(1,61)=5.80 ; \mathrm{p}<0.01, \eta_{\mathrm{p}}{ }^{2}=0.36\right]$ and $\mathrm{a}$ significant main effect of session $[\mathrm{F}(2,122)=67.30 ; \mathrm{p}<0.001$, $\left.\eta_{\mathrm{p}}{ }^{2}=0.53\right]$. There was also a significant group by session interaction $\left[\mathrm{F}(2,122)=2.79 ; \mathrm{p}<0.01, \eta_{\mathrm{p}}{ }^{2}=0.22\right]$.

To further analyze the main effects and interactions, we performed paired t-tests which showed that there were significant improvements in performance at post-training compared to baseline for the $20 \mathrm{~s}[\mathrm{t}(9)=-7.34 ; \mathrm{p}<0.001], 30 \mathrm{~s}[\mathrm{t}(9)=$ $-8.79 ; \mathrm{p}<0.001], 40 \mathrm{~s}[\mathrm{t}(9)=-4.77 ; \mathrm{p}<0.01], 50 \mathrm{~s}[\mathrm{t}(8)=$ $-2.80 ; \mathrm{p}<0.05]$ and 80 year olds $[\mathrm{t}(8)=-3.75 ; \mathrm{p}<0.01]$ but not for the $60 \mathrm{~s}[\mathrm{t}(9)=-1.93 ; \mathrm{p}=0.09]$ or 70 year olds $[\mathrm{t}(9)=-1.44$; $\mathrm{p}=0.18]$. However, examination of baseline versus retention $[\mathrm{t}(9)$ $=-4.40 ; \mathrm{p}<0.01]$ revealed significant improvement from baseline for the seventy year olds, but not for the 60 year olds $[\mathrm{t}(9)=-0.87 ; \mathrm{p}=0.41]$. No differences were found between posttraining and retention in separate tests for each age group apart from the 70 year olds [t $(9)=-2.47 ; \mathrm{p}<0.05]$.

Separate one-way ANOVAS and pairwise comparisons (with Bonferroni corrections applied) on the baseline and post-training data revealed no significant differences between any of the groups at baseline, but a significant difference between the 70 year olds and 20 year olds $[\mathrm{p}<0.01], 30$ year olds and $[\mathrm{p}<0.01] 40$ year olds $[\mathrm{p}<0.05]$ at post-training (in all cases the younger group performing better).

\section{Learning Rates for $90^{\circ}$ Relative Phase}

As well as examining potential differences in the amount of learning between baseline and post-training or retention, we also wanted to determine whether the learning rates between the groups were different and if so, exactly how different. Figure 3 shows the mean learning curve for each of the age groups performing $90^{\circ}$ relative phase across all sessions (apart from retention). Exponential functions were fitted to the data. The functions were of the form:

$$
P T T \equiv a * \exp (-b / s)
$$

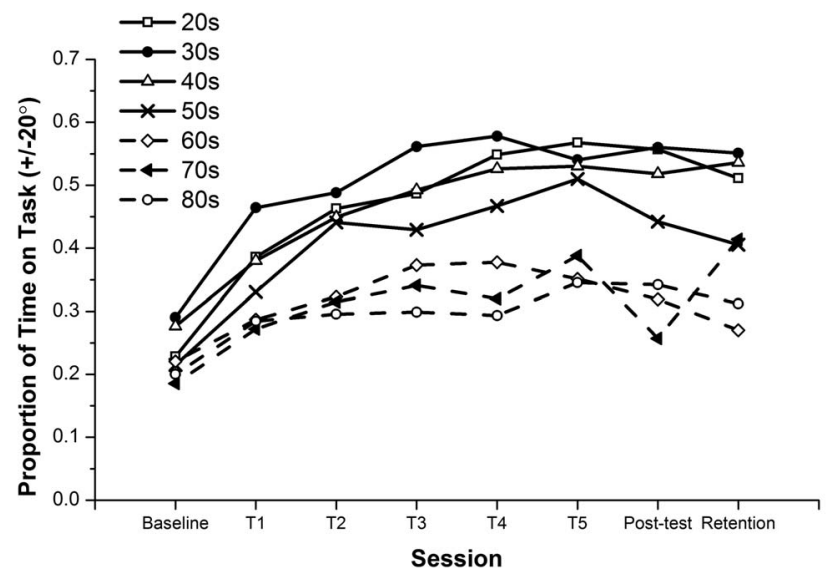

Figure 3. Learning $90^{\circ}$. Proportion of time on task for each age group across all training and assessment sessions. doi:10.1371/journal.pone.0085758.g003 where PTT is 'Proportion of Time on Task', $\mathrm{S}$ is session $(1=$ baseline and $7=$ post-training $)$, and $\mathrm{a}$ and $\mathrm{b}$ are parameters. As mentioned in the methods section, the function was fitted in three different ways and results were compared to be sure they were essentially the same. First, the function was fit to the means separately for each age group using Quasi-Newton estimation in Systat 5.2. This yielded $r^{2}>0.80$ in all cases. The values for parameter a were 0.664 for the $20 \mathrm{~s}$ and $30 \mathrm{~s}, 0.597$ for the $40 \mathrm{~s}$, 0.554 for the $50 \mathrm{~s}, 0.391$ for the $60 \mathrm{~s}, 0.369$ for the $70 \mathrm{~s}$ and 0.360 for the $80 \mathrm{~s}$. For parameter b they were 1.073, 0.750, 0.802. 0.919, $0.556,0.607$ and 0.577 for each group respectively from $20 \mathrm{~s}$ through $80 \mathrm{~s}$. Secondly, the PTT means and session numbers were transformed as follows:

$$
P T T \rightarrow \ln (P T T) \text { and } S \rightarrow 1 / S
$$

Least squares linear regression was used to fit a line to the relation between the two sets of transformed values, again separately for each group. The $\mathrm{r}^{2}$ were $0.98,0.96,0.98,0.90$, $0.56,0.69$, and 0.89 for each group respectively from $20 \mathrm{~s}$ through 80 s. All were significant $\mathrm{p}<0.05$ or better.

Third and finally, transformation and linear regression was used again applied to the combined individual participant data for each group. However, in this last case, we also used multiple linear regression to test differences in slope and intercept between the groups taken two at a time [26]. Both slope and intercept differences yield a difference in learning rate as both form part of the relevant equation, so differences in either are summarised below. The results of the comparisons of $20 \mathrm{~s}$ and all other groups were significant $(p<0.01)$ with differences in slope $(20 \mathrm{~s}$ vs. all groups apart from the $50 \mathrm{~s}$ ) and intercept (20 vs. all groups apart from the $30 \mathrm{~s})$. The $30 \mathrm{~s}$ were significantly different from the $50 \mathrm{~s}$, $60 \mathrm{~s}, 70 \mathrm{~s}$ and $80 \mathrm{~s}$ in terms of intercept $(\mathrm{p}<0.01)$, as were the $40 \mathrm{~s}$ and $50 \mathrm{~s}$ compared to the $60 \mathrm{~s}, 70 \mathrm{~s}$ and $80 \mathrm{~s}$. The three older groups were not significantly different from each other in terms of either slope or intercept.

In the two sets of linear regression analyses (i.e. using means and individual participant data), the resulting linear equations were transformed back into the form of Equation (1). The values found for the parameters $\mathrm{a}$ and $\mathrm{b}$ using all three approaches were essentially the same. Then, in each case, we computed the first derivative of the function in Equation (1), that is:

$$
(a * b) / S^{2} * \exp (-b / S)
$$

We evaluated this derivative at $\mathrm{S}=1$ to derive an estimate of the learning rate. Again, the resulting estimates were nearly identical using all three fitting methods. The resulting learning rates were: $20 \mathrm{~s}=0.243, \quad 30 \mathrm{~s}=0.228, \quad 40 \mathrm{~s}=0.215, \quad 50 \mathrm{~s}=0.203$, $60 \mathrm{~s}=0.125,70 \mathrm{~s}=0.122,80 \mathrm{~s}=0.117$ (reporting the mean of the results of the 3 methods in each case). You can see from Figure 4 that there is a steep drop in learning rate between the $50 \mathrm{~s}$ and $60 \mathrm{~s}$. The learning rates for the 3 older groups were almost exactly half that for young adults in their $20^{\prime} \mathrm{s}$.

\section{$0^{\circ}$ and $180^{\circ}$ Relative Phase}

We wanted to determine if there were any changes in performance for the untrained coordination patterns, $0^{\circ}$ and $180^{\circ}$, as a function of age group and/or training at $90^{\circ}$. Learning 


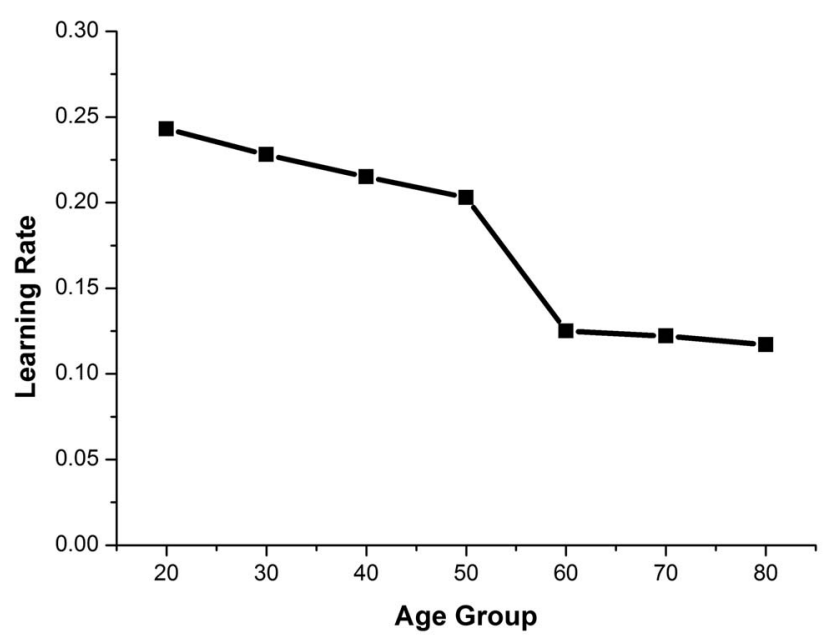

Figure 4. Learning rates for all age groups. Note the dramatic drop from $50 \mathrm{~s}$ to $60 \mathrm{~s}$.

doi:10.1371/journal.pone.0085758.g004

$90^{\circ}$ does not typically transfer to either $0^{\circ}$ or $180^{\circ}$, because learning $90^{\circ}$ entails learning a different perceptual coupling [16].

A repeated measures ANOVA on $0^{\circ}$ performance revealed no significant main effect of group $(\mathrm{p}>0.05)$, but there was a significant main effect of session with performance being higher at post-training (mean time-on-task $=0.69$ ) than baseline (mean time-on-task $=0.66)\left[\mathrm{F}(1,61)=10.78 ; \mathrm{p}<0.01, \eta_{\mathrm{p}}{ }^{2}=0.15\right]$. There was also an interaction between group and session $[\mathrm{F}(6,61)$ $\left.=2.41 ; \mathrm{p}<0.037, \eta_{\mathrm{p}}{ }^{2}=0.19\right]$. To further analyze the main effects and interactions, we performed paired t-tests which showed that there were significant improvements in performance at posttraining compared to baseline for the $60[\mathrm{t}(9)=-2.72 ; \mathrm{p}<0.05]$ and 70 year olds $[\mathrm{t}(9)=-3.01 ; \mathrm{p}<0.05]$, but not any of the other groups $(\mathrm{p}>0.05)$. Separate one-way ANOVAS and pairwise comparisons (with Bonferroni corrections applied) on the baseline and post-training data revealed no significant differences between any of the groups at either baseline or post-test $(\mathrm{p}>0.05)$.

A repeated measures ANOVA on $180^{\circ}$ performance revealed a significant main effect of group $\left[\mathrm{F}(1,61)=3.07 ; \mathrm{p}<0.05, \eta_{\mathrm{p}}{ }^{2}=\right.$ 0.23 with the mean time-on-task of each group as follows: $20 \mathrm{~s}=0.61, \quad 30 \mathrm{~s}=0.57, \quad 40 \mathrm{~s}=0.59, \quad 50 \mathrm{~s}=0.47, \quad 60 \mathrm{~s}=0.48$, $70 \mathrm{~s}=0.45$ and $80 \mathrm{~s}=0.43$. There was no main effect of session or interaction (both $\mathrm{p}>0.05$ ).

Overall therefore, all age groups were equally able to perform $0^{\circ}$ coordination, showing that poorer performance by the older adults in the $90^{\circ}$ condition was not a function of problems using the joystick or seeing the display. However, the older adult groups $(50 \mathrm{~s}, 60 \mathrm{~s}, 70 \mathrm{~s}$ and $80 \mathrm{~s})$ performed $180^{\circ}$ less well than the younger participants $(20 \mathrm{~s}, 30 \mathrm{~s}$, and $40 \mathrm{~s}) .0^{\circ}$ is an easy coordination to maintain because the relative phase is clearly perceived $[13,27]$. The two oscillators move together always in the same direction with no speed difference between them, so that the ability to resolve the phase relation is good throughout the motion $[9,28]$. On the other hand, $180^{\circ}$ yields oppositely directed motion throughout, and the relative speeds of motion vary from zero to a peak at the mid-point of motion. This variation makes relative phase harder to perceive than at $0^{\circ}$. This pattern in the data shows that people over 50 performed worse when the coordination requirements increased in complexity. This suggests that the difference in learning rates at $90^{\circ}$ may be related to the visual perception of coordination, i.e. relative phase.

\section{Discussion}

In a previous study [3] we found that older adults show reduced learning rates when learning a new pattern of coordinated rhythmic movement. The purpose of this study was to extend that finding by examining the performance of all ages across the lifespan from $20 \mathrm{~s}$ through to $80 \mathrm{~s}$ to determine how learning rates change with age. We tested whether adults could learn to produce a novel coordinated rhythmic movement $\left(90^{\circ}\right.$ relative phase) in a visually guided unimanual task. We also determined learning rates to quantify changes in learning with age and to determine at what ages the changes occur. We found, as before, that learning rates of participants in their $70 \mathrm{~s}$ and $80 \mathrm{~s}$ were half those of participants in their $20 \mathrm{~s}$. We also found that learning rates for participants in their $30 \mathrm{~s}$ were significantly greater than rates for participants aged 50 and above. Participants in their $40 \mathrm{~s}$ and $50 \mathrm{~s}$ were faster learners than participants in their $60 \mathrm{~s}, 70 \mathrm{~s}$ and $80 \mathrm{~s}$, and the three oldest groups did not differ from one another in terms of how fast they learned. What was most interesting was the gradual slow decline in learning rate with age until approximately age 50, when there was a sudden drop to a reduced learning rate for the 60 though 80 year olds. The reduced learning rate of the older participants was about half the learning rate of the younger participants.

The mechanisms underlying the reduced learning rates in the 60, 70 and 80 year olds are likely to be multiple, but as we mentioned in our previous paper [3] we think deficits in motion perception are key. If you cannot perceive $90^{\circ}$ how can you be expected to produce it? In addition to examining learning at $90^{\circ}$, we also tested performance of $0^{\circ}$ and $180^{\circ}$ before and after training at $90^{\circ}$. We found that the groups did not differ in their ability to perform $0^{\circ}$, but that the 50, 60, 70 and 80 year olds showed reduced performance at $180^{\circ}$ compared to participants in their $20 \mathrm{~s}, 30 \mathrm{~s}$ and $40 \mathrm{~s}$. According to the Bingham model of rhythmic coordination $[9,10,28]$ the difference in stability of performance between $0^{\circ}$ and $180^{\circ}$ is produced by differences in the relative speeds of the two oscillators (empirically confirmed by Snapp-Childs, et al. [10]). For $0^{\circ}$, the dots move together and the relative speed difference between them is zero. In $180^{\circ}$, the relative speed difference varies over the cycle to be zero at the end points and greatest when the dots pass each other in the middle travelling in opposite directions. Speed differences like these condition the ability to see the relative directions of motion that, according to the model, specify the relative phase. Learning to produce $90^{\circ}$ entails perceptual learning, namely, learning to perceive $90^{\circ}$. This has been shown by Wilson et al. [11] who found that participants were able to perform $90^{\circ}$ once they had learned to see it, without any actual motor practice of the coordinative movements. $90^{\circ}$ is specified by a more complex pattern of speeds and positions, rather than merely relative direction. This more complex spatialtemporal pattern is apparently difficult for older participants to learn to perceive. Further research might investigate whether training older participants to perceive $90^{\circ}$ could compensate for the $50 \mathrm{~s}$ cliff we found in learning how to produce it.

Aging has also been shown to negatively affect a variety of tasks requiring visual perception of motion (see Andersen [29] for a review). For example, visually discriminating speeds is difficult for older adults [30-31] and aging detrimentally affects the ability of older adults to visually perceive the 3 -D shape of objects defined by motion [32-33]. Changes such as these are likely to be underpinned by general changes in cortical function that occur with age, such as decreases in neuronal inhibition leading to reduced centre-surround antagonism in visual cortex and less finely resolved motion detection systems [34-36]. 
Why we found the sudden drop in learning rate at around age 60 remains unclear. Perhaps we go through specific neurological changes at this age, or the behavioural effects of cortical changes are non-linear and at 60 become suddenly more apparent. Interestingly, the fact that learning rates showed a sudden drop at 60 , with the 50 year olds remaining at the top of the metaphorical cliff, was not coincident with the decrement that we found in performance of the $180^{\circ}$ relative phase task, where the 50 year olds showed a reduced performance level relative to younger participants and looked very similar to those in their $60 \mathrm{~s}, 70 \mathrm{~s}$ and $80 \mathrm{~s}$. Perhaps the ability to perform $180^{\circ}$ degrees acts as a marker for subsequent deterioration in learning rate at $90^{\circ}$, as the ability to deal with more complex motion patterns begins to decline.

In conclusion, we have identified a dramatic change in perceptuo-motor learning rates of a standard laboratory task (coordinated rhythmic movement) at around the late $50 \mathrm{~s}$ or early $60 \mathrm{~s}$. This is surprisingly early, and has implications for movement

\section{References}

1. de Rekeneire N, Visser M, Peila R, Nevitt MC, Cauley JA, et al (2003) Is a fall just a fall: correlates of falling in healthy older persons. The health, aging and body composition study. J of Am Geriatr Soc 51: 841-846.

2. Spirduso WW (1995) Issues of quantity and quality of life. In Spirduso WW, editors. Physical dimensions of aging. Champaign, IL: Human Kinetics pp.5-30.

3. Coats RO, Snapp-Childs W, Wilson AD, Bingham GP (2013) Perceptuo-motor learning rate declines by half from 20s to 70/80s. Exp Brain Res 225: 75-84.

4. Kelso JAS (1981) On the oscillatory basis of movement. Bull Psychon Soc 18:63.

5. Zanone PG, Kelso JAS (1992) Evolution of behavioral attractors with learning: Nonequilibrium phase transitions. J Exp Psychol Hum Percept Perform 18(2): 403-421.

6. Swinnen SP, Verschueren SMP, Bogaerts H, Dounskaia N (1998) Age-related deficits in motor learning and differences in feedback processing during the production of a bimanual coordination pattern. Cognitive Neuropsych 15(5): $439-466$.

7. Serrien DJ, Swinnen SP, Stelmach GE (2000) Age-related deterioration of coordinated interlimb behaviour. J Gerontol 55B(5): 295-303.

8. YamanishiJ, Kawato M, Suzuki R (1980) Two coupled oscillators as a model for the coordinated finger tapping by both hands. Biol Cybern 37: 219-225.

9. Bingham GP (2004b) Another timing variable composed of state variables: Phase perception and phase driven oscillators. In: Hecht H, Savelsbergh G (eds) Theories of Time-to-Contact. MIT Press, Boston.

10. Snapp-Childs W, Wilson AD, Bingham GP (2011) The stability of rhythmic movement coordination depends on relative speed: the Bingham model supported. Exp Brain Res 215: 89-100.

11. Wilson AD, Snapp-Childs W, Bingham GP (2010a) Perceptual Learning Immediately Yields New Stable Motor Coordination. J Exp Psychol Hum Percept Perform 36(6): 1508-1514.

12. Wimmers RH, Beek PJ, Wieringen PCW (1992) Phase transitions in rhythmic tracking movements: A case of unilateral coupling. Hum Move Sci 11(1-2): $217-$ 226.

13. Wilson AD, Collins DR, Bingham GP (2005a) Perceptual coupling in rhythmic movement coordination - Stable perception leads to stable action. Exp Brain Res 164: 517-528.

14. Schmidt RC, Carello C, Turvey MT (1990) Phase transitions and critical fluctuations in the visual coordination of rhythmic movements between people. J Exp Psychol Hum Percept Perform 16: 227-247.

15. Temprado JJ, Swinnen SP, Carson RG, Tourment A, Laurent M (2003) Interaction of directional, neuromuscular and egocentric constraints on the stability of preferred bimanual coordination patterns. Hum Move Sci 22: 339 363.

16. Wilson AD, Bingham GP (2008) Identifying the information for the visual perception of relative phase. Percept Psychophys 70: 465-476.

17. Kovacs AJ, Buchanan JJ, Shea CH (2009a) Bimanual 1:1 with 90 degrees continuous relative phase: difficult or easy! Exp Brain Res 193(1): 129-136. rehabilitation following stroke (which disproportionately affects older adults). Research on older adults typically focuses on older age groups (e.g. $60 \mathrm{~s}$ and up); these data suggest that it is vital to take a lifespan view in order to identify earlier, functionally relevant changes.

\section{Supporting Information}

Figure $\mathbf{S 1} \mathbf{9 0}^{\circ}$. Shows $90^{\circ}$ phase relation. (GIF)

\section{Author Contributions}

Conceived and designed the experiments: GPB WSG ROG ADW. Performed the experiments: ROC AJF WSG. Analyzed the data: ROC GPB WSG. Contributed reagents/materials/analysis tools: WSG ADW. Wrote the paper: ROC GPB ADW WSC AJF.

18. Kovacs AJ, Buchanan JJ, Shea CH (2009b) Using scanning trials to assess intrinsic coordination dynamics. Neurosci Lett 455(3): 162-167.

19. Wilson AD, Collins DR, Bingham GP (2005b) Human movement coordination implicates relative direction as the information for relative phase. Exp Brain Res 165: 351-361

20. Wishart LR, Lee TD, Cunningham SJ, Murdoch JE (2002) Age-related differences and the role of augmented visual feedback in learning a bimanual coordination pattern. Acta Psychol 110: 247-263.

21. Pfeiffer E (1975). A short portable mental status questionnaire for the assessment of organic brain deficit in elderly patients. J of Am Geriatr Soc 23(10): 433

22. Brainard DH (1997) The Psychophysics Toolbox. Spat Vis 10: 433-436.

23. Kleiner M, Brainard D, Pelli D (2007) What's new in Psychtoolbox-3? Perception 36: ECVP Abstract Supplement.

24. Pelli DG (1997) The VideoToolbox software for visual psychopysics: transforming numbers into movies. Spat Vis 10: 437-442.

25. Wilson AD, Snapp-Childs W, Coats RO, Bingham GP (2010b) Learning a coordinated rhythmic movement with task-appropriate coordination feedback. Exp Brain Res 205(4): 513-520.

26. Pedhazur EJ (1982) Multiple regression in behavioral research: Explanation and prediction (2nd ed.) New York: Holt, Rinehart and Winston.

27. Wenderoth N, Bock O, Krohn R (2002) Learning a new bimanual coordination pattern is influenced by existing attractors. Motor Control 6(2): 166-82.

28. Bingham GP (2004a) A perceptually driven dynamical model of bimanual rhythmic movement (and phase perception). Ecol Psychol 16(1): 45-53.

29. Anderson GJ (2012) Aging and vision: Changes in function and performance from optics to perception. WIREs Cogn Sci 3(3): 403-410.

30. Norman JF, Ross HE, Hawkes LM, Long JR (2003). Aging and the perception of speed. Perception 32: 85-96.

31. Snowden RJ, Kavanagh E (2006) Motion perception in the ageing visual system: Minimum motion, motion coherence, and speed discrimination thresholds. Perception 35: 9-24.

32. Norman JF, Clayton AM, Shular CF, Thompson SR (2004) Aging and the Perception of Depth and 3-D Shape from Motion Parallax. Psychol Aging 19: 506-514.

33. Norman JF, Dawson TE, Butler AK (2000) The effects of age upon the perception of depth and 3-D shape from differential motion and binocular disparity. Perception 29: 1335-1359.

34. Betts LR, Taylor CP, Sekuler AB, Bennett PJ (2005) Aging reduces centersurround antagonism in visual motion processing. Neuron 45: 361-366.

35. Liang Z, Yang Y, Li G, Zhang J, Wang Y, et al. (2010). Aging affects the direction selectivity of MT cells in rhesus monkeys. Neurobiol of Aging 31(5): 863-873.

36. Nedelko V, Hassaa T, Hamzeib F, Weiller C, Binkofski F (2010) Ageindependent activation in areas of the mirror neuron system during action observation and action imagery. A fMRI study. Restor Neurol Neuros 28: 737747. 